98 Lösel/Bliesener/Fischer/Papst (o. Fn. 41), S. 152.

99 Chuck Palahniuk beschreibt in seinem Roman „Fight Club“, 1996 (1999 verfilmt von David Fincher u.a. mit Brad Pitt), typische Sensation-Seeker. Es treffen sich Männer zu Kämpfen im Keller einer Bar. Diese Art von Geheimloge ist für den Protagonisten die neue Form einer Selbsthilfegruppe.

100 Kunczik/Zipfel, Gewalt und Medien, 2004, S. 51.

101 Bspw. mit Hilfe des deutschen Fragenkatalog von Gniech/Oetting/Brohl, Untersuchungen zur Messung von „Sensation-Seeking“, 1993.

102 Schubert, Gewalt ist eine Lösung. Morgens Polizist, abends Hooligan, 2010.
103 Farin, Von Guten und Mitläufern, online verfügbar unter: http://www.bpb.de/gesellschaft/ kultur/jugendkulturen-in-deutschland/36239/ von-guten-und-mitlaeufern.

104 Lösel/Bliesener/Fischer/Papst (o. Fn. 41), S. 151.

105 Hare, Psychopathy Checklist-Revised (PCLR), 1991 - deutsche Übersetzung von $\mathrm{Ne}$ dopil/Müller-Isberner;2001; vgl. auch Lösel/ Bliesener/Fischer/Papst (o. Fn. 41), S. 113.

106 Göppinger (o. Fn. 85), \$28 Rdn. 46.

107 Von Erlebniskriminalität geht man auch bei Graffiti-Sprayern aus, Neubacher, ZStW 118 (2007), $855 \mathrm{ff}$.

108 Göppinger (o. Fn. 85), $\mathbb{S} 28 \mathrm{Rdn} .46$.
109 Lösel/Bliesener/Fischer/Papst (o. Fn. 41), S. 151.

110 Lösel/Bliesener, MschrKrim 89 (2006), 229 (239).

111 Lösel/Bliesener/Fischer/Papst (o. Fn. 41), S. 151.

112 Von den seit ca. einem Jahr von der Polizei praktizierten sogenannten „mobilen Massenkontrollen“ der Fußballfans auf Bahnhöfen ist daher unbedingt abzuraten (Züge auf dem Weg zu einem Spiel oder zurück werden angehalten und alle Fans zum Aussteigen aufgefordert, identifiziert und fotografiert), vgl. BT-Drucks. 17/8053 v. 2.12.2011.

\title{
Sicherungsverwahrung: Das Bundesverfassungsgericht als Erfüllungsgehilfe eines gehetzten Gesetzgebers?
}

\author{
Michael Bock/Sebastian Sobota
}

\section{Einleitung}

Die jüngere Rechtsprechung des Bundesverfassungsgerichts (BVerfG) zur Sicherungsverwahrung, insbesondere das Grundsatzurteil $^{2}$ vom Mai 2011, ist in der Literatur überwiegend positiv aufgenommen wor$\operatorname{den}^{3}$. Der Einschätzung, es handele sich bei dem Urteil um einen mutigen Befreiungsschlag, der ähnlich herausragend wie die Urteile bspw. zur lebenslangen Freiheitsstrafe oder zur gesetzlichen Grundlage des Strafvollzugs sei ${ }^{4}$, ist jedoch - wie sogleich zu zeigen sein wird - aus mehreren Gründen entschieden zu widersprechen. Vielmehr wecken die jüngeren Urteile und Beschlüsse zur Sicherungsverwahrung die Befürchtung, dass sich das BVerfG dem Druck von Öffentlichkeit und Politik beugt und vor den Karren punitiver Kriminalpolitik ${ }^{5}$ spannen lässt. Es geht um nicht weniger als die Frage, ob Straftäter noch denselben grundrechtlichen Schutz wie die „Normalbevölkerung" genießen.

II. Das sog. „Abstandsgebot“ - das StVollzG als lediglich symbolisches Recht?

Bereits der in der verfassungsgerichtlichen Argumentation zentrale Begriff des Abstandsgebotes, wonach sich die Ausgestaltung des Vollzugs der präventiven Sicherungsverwahrung qualitativ vom Freiheits- entzug durch Strafe unterscheiden müsse ${ }^{6}$, ist problematisch ${ }^{7}$. Mit dem Abstand zum Strafvollzug wählt das $B \operatorname{VerfG}$ nämlich einen falschen Maßstab. Tatsächlich kann es aufgrund der unterschiedlichen Legitimation mit Schuld auf der einen (Strafe) und dem Sonderopfer zugunsten der Allgemeinheit auf der anderen Seite (Sicherungsverwahrung) nicht auf den Abstand des Strafvollzugs gegenüber dem Maßregelvollzug ankommen, wie ihn insoweit die fehlplatzierten und überdies materiell unzureichenden ${ }^{8}$ Regelungen der $\mathbb{S} 129 \mathrm{ff}$. StVollzG nahelegen. Wenn es nämlich bloß auf den Abstand zwischen Straf- und Maßregelvollzug ankäme, wäre die Verfassungsmäßigkeit des Vollzugs der Sicherungsverwahrung bereits ohne jede Verbesserung für die Verwahrten wieder hergestellt, wenn die Bedingungen im Strafvollzug (wieder) restriktiver bzw. resozialisierungsfeindlicher würden ${ }^{9}$.

Maßstab für die Ausgestaltung des Vollzuges der Sicherungsverwahrung muss (nicht nur aus kriminologischer Sicht) vielmehr das Leben in Freiheit sein ${ }^{10}$. Um es mit den Worten des Strafvollzugsgesetzes auszudrücken, muss „das Leben im Vollzug den allgemeinen Lebensverhältnissen [...] angeglichen werden “ ( $\int 3$ Abs. 1 StVollzG). Dies gilt für die Sicherungsverwahrung deshalb umso mehr, weil die Untergebrachten ihre Strafe verbüßt haben und rein präventiv zum Schutz der Allgemeinheit verwahrt werden. Einschränkungen in der Angleichung darf es somit nur aus absolut zwingenden Gründen der Sicherheit (und nicht aus ökonomischen, punitiven oder sonst praktischen Erwägungen heraus) geben ${ }^{11}$. Dies ist auch deshalb geboten, weil das Verhalten in der Unterbringung mit zunehmender Nähe zum Leben in Freiheit an prognostischer Aussagekraft gewinnt ${ }^{12}$.

Zum Widerspruch fordern auch Bewertungen heraus, nach denen das aus dem Abstandsgebot abgeleitete Vollzugskonzept des $B$ VerfG von „großer Sensibilität und Sachkunde“ 13 zeuge. Zusammengefasst lässt sich zwar sagen, die neue Sicherungsverwahrung soll nach dem Willen des $B \operatorname{VerfG}$ therapeutisch und freiheitsorientiert ausgestaltet sein, es soll gerichtlichen Rechtsschutz, Lockerungen sowie Entlassungsvorbereitung geben und es soll auch die Mitwirkungsbereitschaft der Untergebrachten gefördert werden. Doch zum einen scheint ein derart konkreter Gesetzgebungsauftrag durch ein Verfassungsgericht kaum mit dem Gewaltenteilungsprinzip vereinbar ${ }^{14}$. Zum anderen fällt hier sogleich auf, dass die Regelungen, die das $B$ VerfG für eine verfassungskonforme Ausgestaltung der Sicherungsverwahrung fordert, gar keine anderen sind als die des aktuell geltenden (Bundes-) 
Strafvollzugsgesetzes, insbesondere wenn man an den Vollzug der Freiheitsstrafe in sozialtherapeutischen Anstalten denkt:

So lässt sich das vom $B \operatorname{VerfG}$ entwickelte sog. Motivierungsgebot ${ }^{15}$ nahezu wörtlich dem $\int 4$ Abs. 2 S. 2 StVollzG entnehmen. Das sog. Trennungsgebot ${ }^{16}$ beschreibt im Kern nichts anderes als den allgemeinen Angleichungsgrundsatz in $\$ 3$ Abs. 1 StVollzG. Auch sonst findet sich im Urteil eine Reihe von strafvollzugsrechtlichen Forderungen, wie sie der Gesetzgeber im StVollzG längst berücksichtigt hat. Eine Behandlungsuntersuchung ${ }^{17}$, die modernen wissenschaftlichen Anforderungen entspricht, sowie eine entsprechend individuelle Vollzugsplanung sind Selbstverständlichkeiten für einen Strafvollzug, dessen Ziel die Resozialisierung seiner Insassen ist. Ebenso selbstverständlich ist die regelmäßige Überprüfung der Voraussetzungen einer weiteren Unterbringung ${ }^{18}$. Dies gelingt natürlich nur, wenn dem Inhaftierten durch die Gewährung von Lockerungen eine echte Entlassungsvorbereitung und Erprobung zuteil wird. Auch ein funktionierendes Übergangsmanagement ist zwingend erforderlich, um der nötigen Gefährlichkeitsprognose ein konkretes Szenario (insbesondere im Hinblick auf Aufenthalts-, Kontakt- und Leistungsbereich) zugrunde legen zu können.

Ein Abstand zum Strafvollzug ergibt sich durch diese verfassungsgerichtlichen Vorgaben also nur, wenn man eingesteht, dass der Vollzug der Freiheitsstrafe in der Praxis den gesetzlichen Vorgaben der Strafvollzugsgesetze gar nicht entspricht ${ }^{19}$. Das ist mindestens bemerkenswert.

Einzig mit der Forderung, den Untergebrachten einen durchsetzbaren Rechtsanspruch $^{20}$ auf die Vornahme von Maßnahmen einzuräumen, die zur Minderung ihrer Gefährlichkeit erforderlich sind, geht das $B \operatorname{VerfG}$ weiter als die derzeit bestehende Gesetzeslage. Dies ist angesichts einer Praxis $^{21}$, die sich zum Teil als bloßer Verwahrvollzug darstellt, uneingeschränkt zu begrü$B^{22}$. Insgesamt versäumt es das $B \operatorname{VerfG}$ in diesem Zusammenhang aber, positiv zu formulieren, welche weitergehenden Rechte/Ansprüche den Untergebrachten gewährt werden müssen, um ihr alltägliches Leben weniger deprivierend auszugestalten. Wenn man bedenkt, dass nicht wenige Verwahrte als ,untherapierbar“ gelten, wirkt die starke Betonung der therapeutischen Ausrichtung dagegen nicht nur zu optimistisch ${ }^{23}$, sondern macht auch anfällig für Missbrauch dergestalt, dass Straftäter trotz „Unthera- pierbarkeit" zu Therapiezwecken verwahrt werden $^{24}$.

\section{Die „psychische Störung“ ( $\mathbb{1}$ ThUG) - psychisch gestört, aber voll verantwortlich?}

Neben den Vorgaben für die tatsächliche Ausgestaltung des Vollzugs der Sicherungsverwahrung beschäftigt sich das $B \operatorname{VerfG}$ auch mit den rechtlichen Voraussetzungen einer übergangsweisen wie ggfs. neu zu regelnden, verfassungskonformen, nachträglichen Sicherungsverwahrung. Eine rückwirkend angeordnete oder verlängerte Sicherungsverwahrung könne demzufolge nur noch dann als verhältnismäßig angesehen werden, wenn eine hochgradige Gefahr schwerster Gewalt- oder Sexualstraftaten bestehe und eine „psychische Störung“ (\$ 1 ThUG) “ vorliege ${ }^{25}$. Im Zentrum dieser Vorgaben steht somit das Erfordernis einer sog. „psychischen Störung “26, deren Feststellung Ärzten obliegen soll ${ }^{27}$. Hier knüpft das BVerfG an das umstrittene ThUG an ${ }^{28}$. Inzwischen hat es mehrere obergerichtliche Entscheidungen gegeben, in denen Begriff und Anwendung der "psychischen Störung “ konkretisiert wurden ${ }^{29}$. Teilweise stoßen diese Judikate in der Literatur auf (eingeschränkte) Zustimmung ${ }^{30}$. Besonderer Betrachtung bedarf indes die beispiellose Entscheidung der 3. Kammer (sic!) des 2. Senats des $B \operatorname{VerfG}$, in der kürzlich der Begriff der „psychischen Störung“ von den „überkommenen Kategorisierungen der Psychiatrie“ entkoppelt wurde ${ }^{31}$.

\section{Der Beschluss des BVerfG v. 15.09.2011 - 2 BvR 1516/11}

\section{a) Die Verfassungsbeschwerde als Bumerang}

Um die Brisanz dieses Beschlusses einschätzen zu können, ist es notwendig, sich die Ausgangslage des Verfahrens vor Augen zu halten:

Der Verwahrte, der zweifelsohne grausame Verbrechen begangen hat ${ }^{32}$, erreicht mit der Beschwerde vor dem OLG Hamm seine Entlassung aus der Sicherungsverwahrung. Der Senat, der u.a. von einem der erfahrensten forensischen Psychiater Deutschlands sachverständig beraten wird, begründet mit hohem Aufwand, warum der Untergebrachte zwar hochgefährlich, aber eben nicht „psychisch gestört“ sei. Dabei dürfte die Richter ein mulmiges Gefühl beschlichen haben, einen seit Ende der 1970er-Jahre fast durchgängig inhaftierten Schwerverbrecher ohne echte Vorbereitung in die Freiheit zu entlassen. Deshalb schob das OLG Hamm die Entlassung um weitere sechs Monate auf, „um die erforderlichen Entlassvorbereitungen (sic!) treffen zu können“"33. Diese Entscheidung war abschließend und konnte nur noch vom Untergebrachten selbst im Wege der Verfassungsbeschwerde angegriffen werden - einem Verfahren, das seinem individuellen Grundrechtsschutz dienen soll ${ }^{34}$. In diesem guten Glauben erhebt er also die Verfassungsbeschwerde und wendet sich gegen die sechsmonatige Fortdauer, für die - wie er zutreffend vorträgt - keine rechtliche Grundlage existiert. Der Beschwerdeführer und sein Rechtsbeistand dürften aus allen Wolken gefallen sein, als ihnen der Beschluss des $B \operatorname{Verf} G$ zugegangen ist. Darin wird die Verfassungsbeschwerde zwar für „offensichtlich begründet“ ${ }^{35}$ erklärt, im Wege vermeintlich effektiven Grundrechtsschutzes wird jedoch auch der begünstigende Teil des angefochtenen Beschlusses, nämlich die Anordnung der Entlassung aus der Sicherungsverwahrung, gleich mitaufgehoben. Zuvor erteilte das BVerfG nicht einmal einen Hinweis, der dem Beschwerdeführer die Rücknahme seines für ihn nachteiligen Rechtsmittels ermöglicht hätte ${ }^{36}$. Und schließlich gab das Verfassungsgericht im Stile eines Revisionsgerichtes ${ }^{37}$ dem nun wieder zuständigen LG Arnsberg, das zuvor schon einmal rechtswidrig die Fortdauer der Unterbringung anordnete, eine detaillierte Anleitung, wie künftig eine Fortdauer der Sicherungsverwahrung zu begründen sein könnte. Statt zu der angestrebten sofortigen $^{38}$ Entlassung führt die „erfolgreiche“ Verfassungsbeschwerde des Betroffenen nun $\mathrm{zu}$ einem zeitlich unbestimmten Verbleib in der Sicherungsverwahrung. Mit anderen Worten ausgedrückt stellt der Erfolg der Verfassungsbeschwerde den Beschwerdeführer schlechter, als es der Misserfolg getan hätte!

Der rechtsstaatliche Flurschaden, den dieser Beschluss angerichtet hat, ist beträchtlich ${ }^{39}$. Wegen dieser Einführung einer reformatio in peius („Verböserung“) im Verfassungsgerichtsverfahren werden sich Betroffene in Zukunft fragen müssen, ob sie sich nicht lieber mit einer grundrechtsverletzenden Entscheidung abfinden, als das Risiko einzugehen, im Wege einer „erfolgreichen“ Verfassungsbeschwerde letzten Endes noch schlechter gestellt zu werden. Es entsteht der Eindruck, dass die Richter in Antizipation der medialen Reaktion, die solche - aus der Sicht (nicht nur) von Boulevardmedien 
- unpopulären Entscheidungen zugunsten Inhaftierter zeitigen, lieber einen Verfassungsbruch durch das Verfassungsgericht riskieren, als auch den Grundrechten von Schwerverbrechern zur Geltung zu verhelfen. Dazu (und zu den weiteren inhaltlichen Ausführungen des $B$ VerfG) dürfte auch beigetragen haben, dass sich das OLG Hamm mehrfach und explizit auf das grundlegende Urteil des BVerfG beruft ${ }^{40}$. Die Ausführungen des OLG Hamm nicht zu beanstanden, wäre wohl zu Recht als deren Billigung durch das $B \operatorname{Verf} G$ aufgefasst worden und hätte wohl die Pläne des Gesetzgebers durchkreuzt, eine Verwahrung der gewünschten Altfälle zu ermöglichen.

\section{b) Die Psychiatrie als „Büttel der Strafrechtspflege"}

Dies führt sogleich zum ebenso bemerkenswerten obiter dictum des BVerfG, wonach sich die Auslegung des unbestimmten Rechtsbegriffs der „psychischen Störung“ von „den überkommenen Kategorisierungen der Psychiatrie“ lösen müsse und es fortan auf „den Grad der objektiven Beeinträchtigung der Lebensführung in sozialer und ethischer Hinsicht" ankomme, der wiederum „anhand des gesamten - auch des strafrechtlich relevanten - Verhaltens der Betroffenen zu bestimmen" $\mathrm{sei}^{41}$.

Nach der Einführung des ThUG und dem Urteil des BVerfG war anfangs unklar, ob und wie sich eine dritte Kategorie „gestörter" Straftäter überhaupt in das Sanktionensystem des Strafrechts (und ggfs. der landesrechtlichen Unterbringungsgesetze für psychisch Kranke) einfügen lässt ${ }^{42}$. Durch die Pathologisierung der Klientel der neuen nachträglichen Unterbringung wird nämlich der Abstand zur Maßregel des $\$ 63$ StGB verwischt. Waren bisher die in der Sicherungsverwahrung Untergebrachten nach der Vorstellung des Gesetzgebers gerade nicht gestört, sollen sie es jetzt sein, was folgerichtig zu der Frage führt, was sie denn eigentlich von den nach $\$ 63$ StGB Untergebrachten unterscheiden soll. Entweder man ist so „gestört“, dass man „gefährlich“ ist - dann liegen mindestens die Voraussetzungen des $₫ 21 \mathrm{StGB}$ vor und man wird in die Maßregel nach $\ 63$ StGB eingewiesen. Oder man ist nicht gestört, voll schuldfähig und kommt in den Strafvollzug, wo man je nach auffälligen Persönlichkeitsakzentuierungen oder schweren Sozialisationsdefiziten sozialtherapeutisch behandelt wird. Diese Alternative erscheint klar, auch wenn es wegen der Unsicherheit bei der Diagno- se und der unterschiedlichen Handhabung $\operatorname{der} \int \mathbb{S} 20,21$ StGB faktisch zu Überschneidungen kommen mag, so dass es bei vielen Straftätern fraglich scheint, warum sie in der Sozialtherapie und nicht in der forensischen Psychiatrie sitzen (und umgekehrt) ${ }^{43}$. Folgt man dem Gedanken bis hierher, läuft alles auf die weitere Frage hinaus, ob es psychopathologische Störungsbilder gibt, die einerseits so wenig Störung beinhalten, dass $\mathbb{2 1}$ StGB nicht einschlägig ist, andererseits aber so viel Störung aufweisen, dass die sozialtherapeutische Behandlung nicht ausreicht. Der Psychiater Norbert Leygraf hatte auf einer Fachtagung der Kriminologischen Zentralstelle $^{44}$ im ersten Zugriff (sinngemäß) wie folgt differenziert: Es gebe ex lege nun einfach nur „böse“ gefährliche Straftäter, die man freilassen müsse, und solche, die gefährlich und psychisch gestört seien, und deshalb „drin“ bleiben müssen ${ }^{45}$. Diese Unterscheidung ist mit dem jüngsten Verdikt aus Karlsruhe hinfällig. Von nun an ist derjenige (im juristischen Sinne und je nach Bedarf) gestört, der kriminell ist. Und kriminell ist er, weil er gestört ist ${ }^{46}$. Hier bewahrheiten sich sämtliche bösen Vorahnungen ${ }^{47}$ aus den Reihen der Psychiatrie. Die Psychiatrie hat fortan im Bereich der Sicherungsverwahrung/Therapieunterbringung nicht mehr als ein bloßer „Büttel der Strafrechtspflege" zu sein: Es sind zwar weiterhin Fachgutachten zu erstatten, diese sollen dem Gericht aber nicht mehr die nötige Sachkunde zur Beantwortung der Rechtsfrage vermitteln, sondern werden von nun an völlig losgelöst von empirisch belegten Kategorien der Psychiatrie gewürdigt, um die vermeintlich EGMR-/verfassungskonforme Verwahrung der gewünschten Klientel zu ermöglichen. Die juristische Instrumentalisierung der „dissozialen Persönlichkeitsstörung “48 macht es möglich:

\section{Die dissoziale Persönlichkeitsstörung}

Bei dieser Persönlichkeitsstörung bestand bisher Einigkeit, dass sie die Erheblichkeitsschwelle des $\ 21$ StGB regelmäßig nicht erreicht ${ }^{49}$ und diese Persönlichkeitsstörung ist es bekanntlich auch, die Kriminellen in den allermeisten Fällen attestiert wird ${ }^{50}$. Schaut man sich die Merkmale der dissozialen Persönlichkeitsstörung näher an, so drängen sich im Zusammenhang mit den hier anstehenden Überlegungen zwei Fragen besonders dringlich auf:

Zum einen ist es ihre zirkuläre Struktur, die sie dann erhält, wenn sie zur Kriminalpro- gnose eingesetzt wird. Das Verletzen von Regeln und sonstiges unangepasstes Verhalten sind wesentliche Begriffsmerkmale dieser Störung, weshalb sie in einer ärztlichen, pathologisierenden Diktion nicht viel anderes aussagt als der frühere Begriff des „gefährlichen Gewohnheitsverbrechers“ bzw. des „Hangtäters“ ${ }^{51}$. Dies dürfte wohl auch der Grund sein, warum diese Störung bei Straftätern so oft diagnostiziert wird ${ }^{52}$.

Der zweite Aspekt ist der, dass diese Persönlichkeitsstörung kein psychopathologisches Störungsbild im engeren Sinn ist, weil die Funktionsweise des psychischen Apparates voll intakt ist. Gerade deshalb führt die dissoziale Persönlichkeitsstörung (alleine) nie zur Anwendung von $\$ 21$ StGB, und gerade deshalb ist die dissoziale Persönlichkeitsstörung eine Störung, deren Diagnose keineswegs Ärzten vorbehalten ist. Man braucht kein ärztliches, kein psychiatrisches Sonderwissen, um die Voraussetzungen dieser Störung diagnostizieren zu können. Dies alles ist längst bekannt, eigentlich konsentiert und doch schließt sich das $B \operatorname{VerfG}$ nun der Sichtweise des Gesetzgebers an ${ }^{53}$, der bei der Schaffung des ThUG gerade diese Personengruppe im Blick hatte ${ }^{54}$. Bezeichnend ist deshalb der Umstand, dass das BVerfG nicht allgemein von Sachverständigen spricht, die diese Störung festzustellen hätten, sondern ausdrücklich von Ärzten ${ }^{55}$. Es verwundert, wenn diese Anknüpfung an die dissoziale Persönlichkeitsstörung in der Kriminologie z.T. auf Zustimmung ${ }^{56}$ stößt, obwohl sie die o. g. Zwickmühle nicht auflöst, sondern bekräftigt. Denn die Straftäter mit dissozialer Persönlichkeitsstörung gehören aus o.g. Gründen eindeutig auf die Seite der nicht im engeren Sinne Gestörten. Sie erfüllen nicht die Voraussetzungen des $\$ 21$ StGB und sollen daher nach bisheriger Auffassung im normalen Strafvollzug bzw. in der Sozialtherapie resozialisiert werden.

\section{Abstand zu $\int \mathbb{S} 20,21$ StGB?}

So wird die Luft immer dünner und die Frage immer dringlicher, um welche psychopathologischen Störungsbilder es sich eigentlich bei der Klientel für die neue nachträgliche Unterbringung handeln soll. Bei einer potentiell lebenslänglichen Unterbringung stellt es schließlich auch unter dem Aspekt der Rechtssicherheit ein ernst zu nehmendes Problem dar, wenn nicht nur Betroffene nicht vorhersehen können, ob für sie die Voraussetzungen der Verwahrung vorliegen - von den bekannten destruktiven Folgen 
(„Damoklesschwert“) für die Resozialisierung im Strafvollzug einmal abgesehen.

Indirekt macht das Gericht nämlich noch weitere Vorgaben für den Schweregrad dieser psychischen Störung, indem es bei der Prognose zukünftiger „schwerster Gewaltund Sexualverbrechen " 57 - schon diese deliktische Fokussierung stellt weitere und neue Anforderungen - einen solch hohen Grad von Wahrscheinlichkeit fordert, dass diese Prognose eigentlich nur bei Straftätern gestellt werden kann, die ihren Antrieben und Impulsen, unabhängig von ihrem jeweiligen Umfeld, nicht standhalten können. Deshalb tangieren diese Maximalanforderungen an die Wahrscheinlichkeit zukünftiger schwerster Straftaten trotz der an sich unterschiedlichen funktionalen Stellung im Gesamtgefüge des Sanktionenrechts mit innerer Notwendigkeit die Voraussetzungen des $\$ 21 \mathrm{StGB}^{58}$. Unweigerlich kommen hier Störungen der Impulskontrolle ins Spiel, mit oder ohne sexuelle Paraphilien, aber jedenfalls „echte“ psychopathologische Störungsbilder. Also sind auch diese zusätzlichen Vorgaben im Urteil des BVerfG geeignet, den Abstand zu den Eingangskriterien der Maßregel nach $\$ 63$ StGB einzuebnen. Daraus folgt, dass es für die vom Gesetzgeber geschaffene und vom $B$ VerfG gebilligte neue Kategorie gestörter Straftäter keinen Raum gibt. Als eine - verfassungsrechtlich sicher hochproblematische - Auffanglösung für die Fälle, in denen sich in der Sozialtherapie herausstellt, dass die Entscheidung für die Anwendung des $\mathbb{2 1}$ StGB falsch war, oder dass die Sozialtherapie nicht ausreicht, oder dass durch die Inhaftierung selbst nicht beherrschbare Störungsbilder entstanden sind, käme allenfalls eine Veränderung der Vorschrift des $\$$ 67a StGB in Betracht, so dass nach Endstrafe eine Überstellung in die Maßregel nach $\ 63$ StGB die logische Konsequenz wäre.

\section{Die Prognose als „taugliche Entscheidungsgrundlage “59}

Fast immer, wenn es um die Sicherungsverwahrung geht, wird auf die Schwierigkeit hingewiesen, zuverlässige Kriminalprognosen zu erstellen ${ }^{60}$. Echte Konsequenzen werden aus diesem Einwand jedoch selten ${ }^{61}$ und wenn, dann allenfalls im Sinne einer allgemeinen Warnung ${ }^{62}$, mit der Anwendung der Sicherungsverwahrung zurückhaltend umzugehen, gezogen. Und so überrascht es auch nicht, dass das BVerfG diesen neuralgischen Punkt der Sicherungsverwahrung in seiner neuesten Entscheidung nahezu voll- ständig ausspart. Lediglich zur Frage, ob eine Prognose bei Jugendlichen und Heranwachsenden per se ausgeschlossen sei, lässt das wiederum sachverständig beratene Gericht verlauten, dass die Gefährlichkeitsprognose auch in diesen Fällen eine „taugliche Grundlage "63 bilde. Dies gelte insbesondere deshalb, weil bestimmte psychische Störungen - namentliche schwere sexuelle Devianzen - bereits in relativ jungem Alter diagnostizierbar seien ${ }^{64}$. Hier verwundert erneut, dass das B VerfG in diesen Fällen keine Überschneidung mit den $\mathbb{\$} \mathbb{S} 20,21$ StGB und folglich $\$ 63 \mathrm{StGB}$ sieht.

\section{BVerfGE 109, 133 im Lackmustest empirischer Forschung}

Im Übrigen kann daraus nur geschlossen werden, dass das $B \operatorname{VerfG}$ insoweit an seinem Urteil von 2004 festhalten möchte ${ }^{65}$. Zur Prognosesicherheit hieß es damals nach mündlicher Anhörung zweier psychiatrischer Sachverständiger:

„Die Unsicherheiten der Prognose, die Grundlage der Unterbringung ist (), haben Auswirkungen auf die Mindestanforderungen an Prognosegutachten und deren Bewertung im Zusammenhang mit dem Übermaßverbot (), beseitigen aber weder die Eignung noch die Erforderlichkeit des Freiheitseingriffs. Prognoseentscheidungen bergen stets das Risiko der Fehlprognose, sind im Recht aber gleichwobl unumgänglich. Die Prognose ist und bleibt als Grundlage jeder Gefahrenabwehr unverzichtbar, mag sie auch im Einzelfall unzulänglich sein. In der Praxis der forensischen Psychiatrie hat sich im Übrigen das Wissen um die Risikofaktoren in den letzten Jahren erheblich verbessert, so dass über einen Teil der Delinquenten relativ gute und zuverlässige prognostische Aussagen gemacht werden können (). Beide in der mündlichen Verhandlung angehörten Sachverständigen haben übereinstimmend angegeben, ein bestimmter und bestimmbarer Anteil der Probanden versammele eine derartige Häufung von Risikofaktoren auf sich, dass eine Gefahr sicher prognostiziert werden könne. Auch wenn der Anteil relativ sicherer Prognosen von den sachverständigen Auskunftspersonen unterschiedlich hoch angesehen wird, bildet die Prognose gerade für die seltenen Fälle hochgradiger Gefährlichkeit, die $\int 67 d$ Abs. 3 StGB im Auge hat, eine taugliche Entscheidungsgrundlage" ${ }^{\text {"66 }}$.

Einmal abgesehen von der fragwürdigen Festlegung auf die Psychiatrie als für die kriminalprognostische Begutachtung von voll schuldfähigen Straftätern zuständige Wissenschaft ${ }^{67}$ überging das Gericht schon damals die methodische Krux der Kriminalprognose: Zwar existiert dank großangelegter Rückfalluntersuchungen inzwischen ein breites Wissen über Risikofaktoren, doch dieses Wissen beschreibt nur eine Gruppe und kann deshalb im Einzelfall nur begrenzt fruchtbar gemacht werden. Gerade Nedopil ${ }^{68}$, dessen Aufsatz das Gericht als Beleg für seine Behauptung zitiert, schränkt dort die Bedeutung empirischen Wissens für die Individualprognose sogleich wieder ein, indem er zu Recht betont, dass „eine weitere Unzulänglichkeit bei der Prognosebeurteilung in der Anwendung empirischen Wissens auf einen Einzelfall liegt" ${ }^{\text {69 }}$. Zum einen nennt er Beispiele, in denen es trotz einer geringen Anzahl von Risikofaktoren zu Rückfällen gekommen ist. Zum anderen erinnert er an Fälle, in denen sich trotz einer massiven Häufung von Risikofaktoren keine neuen Straftaten mehr ereignet haben $^{70}$. Die Behauptung des BVerfG, dass „ein bestimmter und bestimmbarer Anteil der Probanden eine derartige Häufung von Risikofaktoren auf sich versammele, dass eine Gefahr sicher (!) prognostiziert werden könne", ist empirisch nicht haltbar. Denn diese sichere Vorhersage bei einigen wenigen gelingt zwangsläufig nur auf Kosten einer Mehrheit, die falsch-positiv als gefährlich eingestuft wird (hohe Sensitivität bei geringer Spezifität) ${ }^{71}$. Jüngste kriminologische Untersuchungen zeigen, dass die Prognosesicherheit selbst bei schwersten Straftätern katastrophal ist $^{72}$. Diese Erkenntnisse haben sich inzwischen auch in der Literatur herumgesprochen ${ }^{73}$.

$\mathrm{Zu}$ fordern wäre deshalb auch vom $B \operatorname{VerfG}$ eine detaillierte Auseinandersetzung mit Befunden wie von M. Alex, in dessen hochselektiver Stichprobe von 77 vermeintlich hochgefährlichen Straftätern, die aus Rechtsgründen ${ }^{74}$ dennoch freigelassen werden mussten, in den ersten sechs Monaten nach der Entlassung kein einziger ${ }^{75}$ in der von $\$ 66 \mathrm{~b}$ StGB geforderten Weise ${ }^{76}$ rückfällig wurde. Wer ein halbes Jahr nicht für ausreichend hält, muss zur Kenntnis nehmen, dass bei M. Alex auch nach durchschnittlich 33 Monaten Beobachtungszeitraum noch 50 von 77 Probanden (entspricht ca. $65 \%$ ) ohne jeden Rückfall geblieben sind und es in seiner Gruppe insgesamt nur zu vier Katalogtaten gekommen ist. An dieser Stelle ist noch einmal darauf hinzuweisen, dass eine seriöse Entlassungsprognose niemals Gültigkeit für einen längeren Zeit- 
raum beanspruchen kann, da sie sich sonst notwendigerweise auf situative Umstände beziehen muss, die zum Zeitpunkt der Prognosestellung noch gar nicht bekannt sein und daher niemals einen Prognosefehler begründen können ${ }^{77}$. Weil das Recht auch von einem Sachverständigen nichts Unmögliches verlangen darf, verlangt das BVerfG eine zum Zeitpunkt der Entlassung ,gegenwärtige "Gefährlichkeit des zu Verwahrenden, während ein mittel- oder langfristiges Risiko nicht ausreiche. ${ }^{78}$ Von einer solchen kann aber bei einem Rückfall, der sich erst Jahre nach der Entlassung ereignet, nicht mehr gesprochen werden ${ }^{79}$. In diesen Fällen ist die neue Tat nicht aus der Prognose ableitbar, sondern ein zufälliger Treffer, der somit die zuvor diagnostizierte Gefährlichkeit gerade nicht bestätigt ${ }^{80}$. Dem strukturellen Problem unsicherer Kriminalprognosen helfen auch Vorschläge wie eine interdisziplinär besetzte Fachkommission zur Begutachtung gefährlicher Straftäter nicht $a b^{81}$.

\section{Abwägung oder Verwirkung?}

Die nun vom $B$ VerfG geforderte „hochgradige Gefahr schwerster Gewalt- und Sexualstraftaten " 82 ist lediglich eine sprachliche (und deliktsspezifische) Steigerung der „hohen Wahrscheinlichkeit erheblicher Straftaten“ ( $\mathbb{S} 66$ b S. 1 Nr. 2 StGB a.F.). Doch keine der beiden ist in der Praxis auch nur annähernd sicher festzustellen, weshalb es sich bei diesen Begriffen um nicht mehr als eine gesetzgeberische wie verfassungsgerichtliche Fiktion handelt, mit deren Hilfe man der unangenehmen (Abwägungs-) Frage entgehen will, wie viele tatsächlich „ungefährliche“ Menschen man einsperren darf, damit ein paar der tatsächlichen Rückfalltäter „erwischt“ werden ${ }^{83}$. Indirekt und bezüglich der o.g. Prämissen inkonsequent positioniert sich das BVerfG in dieser Frage allerdings schon, wenn es lapidar festhält: „Die Prognose ist und bleibt als Grundlage jeder Gefahrenabwehr unverzichtbar, mag sie auch im Einzelfall unzulänglich sein " ${ }^{84}$. Bei strafrechtlichen Sanktionen geht es aber immer um „Einzelfälle“ ${ }^{* 85}$. Hier entsteht der Eindruck, dass unter dem Deckmantel der angeblichen Prognosesicherheit doch ein Eingriff in die unantastbare Menschenwürde stattfindet, weil die trotz (nicht zutreffend erkannter) Ungefährlichkeit Inhaftierten zum bloßen Objekt der Straftatenbekämpfung/-verhinderung gemacht werden ${ }^{86}$. Nicht minder problematisch, aber insofern ehrlicher erscheint der Ansatz, den Untergebrachten aufgrund ihrer massiven Vorstrafen einen Teil des grund- rechtlichen Schutzes abzusprechen mit der Folge, dass sie das Risiko falsch-positiver Gefährlichkeitsprognosen tragen müssen ${ }^{87}$. Allerdings ist dies nur möglich, wenn man den o.g. Verstoß gegen die Menschenwürde negiert, denn seine Würde kann ein Mensch niemals verwirken ${ }^{88}$. Weitere deliktische Fokussierungen, wie sie etwa in der Literatur vorgeschlagen werden ${ }^{89}$, helfen dem grundsätzlichen Problem dagegen nicht ab. Dies gilt insbesondere deshalb, weil bspw. ein sadistisch oder sexuell motivierter Mörder in der Regel zu lebenslanger Haft verurteilt werden oder unter den Voraussetzungen der $\int \mathbb{S} 20,21,63$ StGB potentiell lebenslänglich untergebracht wird. Hier stellt sich schon rein logisch die Frage nach der Sicherungsverwahrung nicht, weil die (vorzeitige) Entlassung aus der lebenslangen Haft nach $\mathbb{S} \int 57,57 \mathrm{a}$ StGB (ebenso wie die Entlassung aus dem psychiatrischen Krankenhaus, \ $67 \mathrm{~d}$ StGB) eine positive Legalprognose voraussetzt $^{90}$, während die Sicherungsverwahrung das exakte Gegenteil, nämlich eine extrem negative Prognose, verlangt ${ }^{91}$.

\section{Fazit und Ausblick}

Ein eleganter Ausweg aus dem Dilemma, wie eine freiheitlich-demokratische Gesellschaft mit rückfallgefährdeten Schwerverbrechern umgehen soll, ist bislang nicht in Sicht. Einige Grundannahmen sollten aber berücksichtigt werden:

Erkennt man die Unmöglichkeit auch nur halbwegs verlässlicher Gefährlichkeitsprognosen an, tun sich Abgründe auf, die das System der Maßregeln grundsätzlich in Frage stellen. Eine Alternative (jedenfalls zur Sicherungsverwahrung) stellte letztlich nur die Verhängung höherer Strafen dar (Strafrahmen/Strafniveau), allerdings nicht mit dem Ziel, Straftäter allgemein länger wegzusperren. Vielmehr spricht für diesen Weg, dass zum Zeitpunkt der Tat und bei Verurteilung Rechtsklarheit besteht. Es existierte auch keinerlei Notwendigkeit mehr, im Nachhinein mit fragwürdigen Konstruktionen („neue Tatsachen“, „psychische Störung“) eine unbefristete Verwahrung zu verhängen und auf diese Weise in das Vertrauen des Betroffenen einzugreifen. Gleichzeitig müsste die Möglichkeit der vorzeitigen Entlassung massiv ausgebaut werden, um die Betroffenen zu Therapie und Mitarbeit im Vollzug zu motivieren und unnötig lange Strafzeiten zu vermeiden. Darüber hinaus bliebe nach dieser Lösung auch bei Entlassung aus dem Strafvollzug ein erheblicher Strafrest bestehen, der (neben den Instrumenten der Füh- rungsaufsicht) Fehlverhalten während der Bewährungszeit entgegenwirken könnte. Die günstigen Prognosen, die eine Entlassung zwingend voraussetzen würde, sind bekanntlich schon aufgrund der hohen Basisrate positiver Verläufe, insbesondere bei älteren Strafgefangenen ${ }^{92}$, wesentlich treffgenauer als die negativen. Zudem macht es einen erheblichen Unterschied, ob eine naturgemäß unsichere Prognose als Grundlage der schärfsten Sanktion des StGB dienen soll oder bloß zur Beantwortung der Frage, ob eine vorzeitige Entlassung „unter Berücksichtigung des Sicherheitsinteresses der Allgemeinheit verantwortet " ( $\$ 57 \mathrm{StGB})$ werden kann, herangezogen wird. Doch auch die Erhöhung des Strafniveaus bereitete schwierige Probleme, beträfe sie doch notwendigerweise das gesamte System der Freiheitsstrafe, einschließlich ihrer Höchstdauer ( $\int 38$ Abs. 2 StGB) sowie ihr Verhältnis zur lebenslangen Freiheitsstrafe und deren Mindestverbüßungsdauer ( $\int 57$ a Abs. 1 Nr. 1 StGB) $)^{93}$.

Die Zulässigkeit einer sog. Sicherungsstrafe, bei deren Bemessung zusätzlich zum Schuldausgleich negativ-spezialpräventiven Gründen Raum gelassen wird, ist dagegen verfassungsrechtlich zweifelhaft ${ }^{94}$. Zudem entkäme man mit dieser Lösung nicht dem o.g. Prognosedilemma, weil zur Bemessung des aus präventiven Gründen gebotenen Strafanteils wiederum die „Gefährlichkeit“ des Täters bestimmt werden müsste.

All dies sind ohne Zweifel grundsätzliche Fragen, die der Gesetzgeber beantworten muss, weshalb es umso bedauerlicher erscheint, dass das Verfassungsgericht notdürftige Reparaturen an einem prekären System vornimmt, anstatt dem Parlament im Wege der Nichtigerklärung eine Totalreform aufzugeben. So steht am Ende dieser Überlegungen die Erkenntnis, dass die Rechtsprechung des BVerfG nur einen scheinbaren Fortschritt in der Entwicklung eines rechtsstaatlich vertretbaren Umgangs mit Schwerverbrechern darstellt. Obwohl der zuletzt ausufernden ${ }^{95}$ Praxis der Sicherungsverwahrung zu Recht Einhalt geboten wurde, ebnet das Gericht den Weg dafür, dass in Zukunft mit der Konstruktion der „psychischen Störung “ weiterhin eine menschenrechtswidrige Verwahrung betrieben werden wird. Wieder einmal verpasst es das $B \operatorname{VerfG}$, in der Verhältnismäßigkeitsfrage Farbe zu bekennen. Der Bundestag wird nun - kurz nach der jüngsten „Flickschusterei “96 aus dem Jahr 2010 und unter den erschwerten Bedingungen der Landesgesetz- 
gebungskompetenz für den Vollzug - wohl wieder bloß kosmetische Korrekturen am krankenden Konzept der Sicherungsverwahrung vornehmen, indem er lediglich das Urteil der Verfassungsrichter in Gesetzesform gießt ${ }^{97}$. Nicht einmal dass das $B \operatorname{Verf} G$ einen offenkundigen „Etikettenschwindel“ 98 wie das ThUG verwerfen wird, ist angesichts der jüngsten Urteile wahrscheinlich. Hier bleibt letzten Endes nur zu hoffen, dass die Straßburger Richter am EGMR sich nicht von der freundlichen ${ }^{99}$ Rhetorik des BVerfG blenden lassen, sondern die offensichtliche Umgehung ihrer Rechtsprechung erkennen und ahnden werden.

\section{Fußnoten:}

1 Prof. Dr. Dr. Michael Bock ist Inhaber des Lehrstuhls für Kriminologie, Jugendstrafrecht, Strafvollzug und Strafrecht an der Johannes Gutenberg-Universität Mainz; Sebastian Sobota ist wissenschaftlicher Mitarbeiter am Lehrstuhl für Strafrecht und Strafprozessrecht von Prof. Dr. Volker Erb, ebenfalls Mainz, und freier Mitarbeiter am Lehrstuhl von Prof. Bock.

2 BVerfGE 128, 326 = NJW 2011, 1931 = NStZ 2011, 450.

3 Peglau, NJW 2011, 1924; Hörnle, NStZ 2011, 488; Sachs, JuS 2011, 854; Kreuzer/Bartsch u. Eisenberg, StV 2011, 472 u. 480; Streng, JZ 2011; 827, Volkmann, JZ 2011, 835; Landau, NStZ 2011, 537; Zabel, JR 2011, 467; Schöch, GA 2012, 14. Kritisch dagegen die umfassende Kommentierung zu den aktuellen Vorschriften über die Sicherungsverwahrung von RiBGH Eschelbach in Matt/Renzikoswki, StGB-Kommentar, erscheint demnächst bei Vahlen.

4 Schöch, GA 2012, 14-31, 14.

5 Eingehend zum Vormarsch ausschließender Kriminalpolitik Göppinger-Schneider, Kriminologie, 6. Auflage 2008, $\$ 30$.

6 BVerfGE 128, 326, 374 ff.

7 Krit. auch Schöch, GA 2012, 14, 18.

8 So auch Schöch, GA 2012, 14, 18.

9 Ähnlich Streng, JZ 2011, 828, 831. Exemplarisch dazu OLG Nürnberg, StV 2011, 694, wonach das Abstandsgebot in Bayern dazu führen könnte, dass entgegen der üblichen Praxis im Strafvollzug den Sicherungsverwahrten immerhin der Besitz einer Spielekonsole genehmigt wird.

10 Befremdlich ist deshalb die öffentliche Debatte, ob $20 \mathrm{~m}^{2}$ Wohnraum plus Bad und Küchenzeile für Sicherungsverwahrte "Luxus" sei. S. becklink 1017567 und Süddeutsche u.a. v. 07.12.2011. Zum Vergleich: Alleinstehenden Hartz IV-Empfängern stehen nach der Rechtsprechung des BSG 45-50 $\mathrm{m}^{2}$ Wohnraum zu, s. nur BSG, NZS 2009, 338 u. BSG, NZS 2010, 640.

11 Anders als das OLG Nürnberg in Fn. 9: OLG Frankfurt a. M., Beschluss vom 20.03.2012 - 3 Ws 1009/11 (StVollz) = BeckRS 2012, 07913, wo das Verbot einer Spielekonsole für Sicherungsverwahrte u.a. mit der Gefahr schwerer Gewalt- und Sexualstraftaten begründet wird.

12 Dabei scheinen sog. „Longstay“-Einrichtungen, wie sie beispielsweise in den Niederlanden zur Verwahrung gefährlicher Straftäter eingerichtet wurden, nicht mit dem Grundgesetz vereinbar zu sein, vgl. Mushoff, NK 2008, 67.

13 Schöch, GA 2012, 14, 18.

14 Ebenso noch BVerfGE 109, 133, 167.

15 BVerfGE 128, 326, 380.

16 BVerfGE 128, 326, 380 .

17 Vgl. $\$ 6$ StVollzG.
18 BVerfGE 128, 326, 382. Die jährliche Überprüfung birgt allerdings die naheliegende Gefahr einer „Fortschreibeautomatik“, da in diesem - für Maßregelverhältnisse - kurzen Abstand selten wesentliche Fortschritte zu verzeichnen sein werden. Kritisch deshalb auch zu Recht Kreuzer/Bartsch StV, 472, 478. Erschütternde Fallbeispiele bei Leygraf/Leygraf, FPPK 2011, 184.

19 So auch Kreuzer/Bartsch, StV, 472, 479. Ähnlich Streng, JZ 2011, 827, 831.

20 BVerfGE 128, 326, 382.

21 Der Verf. Bock war als forensischer Gutachter in einem der ersten Fälle nach $\mathbb{} 7$ Abs. 2 JGG, über den auch das $B \operatorname{VerfG}$ aaO zu entscheiden hatte, tätig und konnte sich auf diese Weise selbst ein Bild von der therapeutischen Unzulänglichkeit des Strafvollzugs an gefährlichen Straftätern machen. Vgl. zu diesem Fall auch Brettel, ZRP 2010, 121.

22 Zustimmend auch Kreuzer/Bartsch, StV 2011, 472, 478.

23 S. a. Peglau, NJW 2011, 1924, 1925; Streng, JZ 2011, 828, 831; Hörnle, NStZ 2011, 488, 493.

24 Siehe exemplarisch das Gesetz zur Therapierung und Unterbringung psychisch gestörter Gewalttäter (Therapieunterbringungsgesetz ThUG), speziell BT-Dr 17/3403, S. 87 zu $\$ 2$ ThUG. wonach fehlende „Therapierbarkeit“ die Eignung für die Therapieunterbringung nicht beseitige; vgl. Kinzig, NJW 2011, 177, 182.

25 BVerfGE 128, 326, 399 ff.

26 S. jüngst etwa Höffler/Stadtland, StV 2012, 239; Morgenstern, ZIS 2011, 974; Krehl, StV 2012, 27, 29, Streng, JZ 2011, 827, 832.

27 BVerfGE 128, 326, 373.

28 S. etwa die Stellungnahmen vor dem Rechtsausschuss von Kinzig, S. 19 ff., u. N. Leygraf, S. 5 ff., abrufbar im Volltext unter www.bundestag.de; Kreuzer, StV 2011, 122, 124 f., 131; nochmals Kinzig, NJW 2011, 177, 182.

29 So hat der 5. Strafsenat des BGH judiziert, dass die „psychische Störung“ die Schuldfähigkeit nicht einschränken müsse, BGH, NStZ 2011, 453. Derselbe Senat entschied in einem weiteren Verfahren „durch“ und verwarf die Revision des Angeklagten, indem er das Vorliegen einer solchen Störung - ohne Zurückverweisung und obwohl das Landgericht Potsdam zum Urteilszeitpunkt den neuen verfassungsrechtlichen Prüfungsmaßstab noch gar nicht berücksichtigen konnte - kurzerhand selbst bejahte: BGH, StV 2011, 631.

30 Schöch, GA 2012, 14, 27.

31 BVerfG, Beschluss v. 15.09.2011 - 2 BvR 1516/11, gekürzt abgedruckt in StV 2012, 25, 27 m. krit. Anm. Krehl.

$32 \mathrm{Vgl}$. die Ausführungen zu seiner Legalbiographie im Beschluss des OLG Hamm v. 09.06.2011, Az. III-4 Ws 207/10 Rn. 11 ff., im Internet abrufbar unter http://www.justiz.nrw. de.

33 OLG Hamm, Beschluss v. 09.06.2011, Az. III 4 Ws 207/10, Rn. 72

34 Maunz-Dürig, Art. 93 GG Rn. 65 u. Maunz/ Schmidt-Bleibtreu/Klein/Bethge $\mathbb{} 90$ BVerfGG Rn. 1.

35 BVerfG, Beschluss v. 15.09.2011 - 2 BvR 1516/11 Rn. 22.

36 Krehl, StV 2012, 27, 28.

37 Krit. dazu auch Krehl, StV, 27, 29.

38 Zum Zeitpunkt der Kammerentscheidung hätte der Beschwerdeführer noch ca. drei Monate Unterbringung „gespart“...

39 Der Kritik Krehls in StV 2012, 27 ist insoweit uneingeschränkt zuzustimmen.

40 OLG Hamm, Beschluss v. 09.06.2011, Az. III-4 Ws 207/10, Rn. 33 ff., 36.

41 BVerfG, StV 2012, 25, 27.
42 Streng, JZ 2011, 827, 832; Morgenstern, ZIS 2011, 974, 978.

43 S. $\$ \int 67 \mathrm{a}$ u. $67 \mathrm{~d}$ StGB.

44 „Psychologisch-psychiatrische Begutachtung in der Strafjustiz", 06.-07. Juni 2011 im Landeshaus zu Wiesbaden. S. http://www.krimz.de/ tagung11.html.

45 Ebenso Hörnle, NStZ 2011, 488, 491.

46 Vgl. zur zirkulären Argumentation bei der dissozialen Persönlichkeitsstörung auch das Zitat aus den Ausführungen des Sachverständigen im Beschluss des OLG Hamm v. 09.06.2011, Az. III - 4 Ws 207/10, Rn. 46 ff., 60.

47 Siehe insbesondere N. Leygraf, Stellungnahme vor dem Rechtsausschuss, abrufbar unter www. bundestag.de, S. 5 f: „Dies ist der Versuch, die Psychiatrie als Ersatzreserve für das Strafrecht zu nutzen, um den Freiheitsentzug von Menschen zu begründen, die bis dahin explizit nicht als Adressaten psychiatrischer Fürsorge gegolten haben."

48 ICD-10 F60.2 bzw. DSM-IV 301.7.

49 MüKo-Streng $\mathbb{S} 20$ StGB Rn. 93 ff.; Lackner/ Kühl, $\mathbb{2 0}$ StGB Rn. 11.

50 Frädrich/Pfäfflin, R\&P 2000, 95; Marneros/ Ullrich/Rössner, Angeklagte Straftäter. Das Dilemma der Begutachtung, Nomos 2002, S. 70 ff.; Rössner/Marneros/Ullrich, FS Schreiber, S. 387 ff.; Ein Überblick über mehrere Untersuchungen findet sich bei Nedopil, Forensische Psychiatrie (3. Aufl.), Stuttgart u.a. 2007, S. 188.

51 Siehe dazu das beißende Editorial von Kröber zu StV 01/2012, der ein Comeback des Hanges als „psychische Störung“ befürchtet. Zuvor schon in dieselbe Richtung weisend Streng, JZ 2011, 827, 832. Vgl. auch Fn. 46.

52 Nach MüKo-Streng $\$ 20$ StGB Rn. 96 beschreibt die dissoziale Persönlichkeitsstörung nichts anderes als den „Idealtypus des Kriminellen".

53 BVerfGE 128, 326, 394-399.

54 Amtl. Begründung BT-Drs. 17/3404, S. 54.

55 BVerfGE 128, 326, 373 mit Verweis auf BVerfGE 109, 133, 164. Damit korrespondierend $\mathbb{} 9$ Abs. 1 ThUG sowie die Begründung zu $\mathbb{8} 8$ Abs. 1 ThUG, wie Fn. 54, S. 57.

56 Schöch, GA 2012, 14, 26.

57 BVerfGE 128, 326, 399.

58 Nußstein in NJW 2011, 1194, 1196 sieht als Vors. RiLG einer nach $\mathbb{} 4$ Abs. 1 ThUG zuständigen Zivilkammer (!) deshalb die größte praktische Schwierigkeit bei der rechtssicheren Eingrenzung der „psychischen Störung “, die einerseits „Minus zur psychischen Erkrankung“ sein soll, andererseits aber so schwer wiegen muss, dass sie für die hohe Gefährlichkeit kausal ist. S.a. Kinzig, NJW 2011, 177, 182. Angesichts der hohen Prävalenz psychischer Störungen auch in der Normalbevölkerung versteht sich schließlich von selbst, dass psychische Störungen nicht allgemein Kriminalität bedingen.

59 BVerfGE 109, 133, 158.

60 Vgl. statt vieler: Kinzig, Die Sicherungsverwahrung auf dem Prüfstand, Diss. Freiburg 1996, S. 79-98.

61 In diesem Sinne auch Streng, JZ 2011, 827, 828.

62 Eine Mahnung, die angesichts des atemberaubenden Anstiegs der Zahl der Sicherungsverwahrten seit 1998 getrost als wirkungslos bezeichnet werden darf. Vgl. Kinzig, NJW 2011, 177.

63 BVerfGE 128, 326, 373.

64 Der Verf. Bock hat kürzlich als forensischer Gutachter in einem weiteren Verfahren nach $\int 7$ Abs. 2 JGG erlebt, wie sich eine im Heranwachsenden-Alter ärztlich diagnostizierte „kombinierte Persönlichkeitsstörung“, in die sich Züge aus der schizoiden, der emotional instabilen sowie der narzisstischen Form und 
antisozial/dissoziale Züge mischten, nur wenige Jahre später plötzlich abklingen kann. S. zu diesem Verfahren die Anmerkung von Sobota in ZJJ 02/2012, 211.

65 So auch Schöch, GA 2012, 14, 30 und Streng, JZ 2011, 827, 828.

66 BVerfGE 109, 133, 158.

67 Ausführlicher noch BVerfGE 109, 133, 164 ff. Zur Kritik an der klinischen Kriminalprognose Schneider, StV 2006, 99; Bock, StV 2007, 269; ders. in FS Ebert, S. 459, 469 u. FS Heinz, S. 609-620.

68 Nedopil, NStZ 2002, 344.

69 Nedopil, NStZ 2002, 344, 347 f. Überdies fehle es an dieser empirischen Basis für die Gruppe der über 55-Jährigen. Gerade um diese Gefangenenpopulation geht es aber bei Fragen der (nachträglichen) Sicherungsverwahrung, s. dazu Dessecker „Lebenslange Freiheitsstrafe und Sicherungsverwahrung", KrimZ 2011, S. 26 (abrufbar unter http://www.krimz.de/index.php?id=texte).

70 Nedopil erwähnt sogar, dass es in drei von vier Fällen, in denen er selbst eine negative Prognose gestellt hatte und die Straftäter dennoch entlassen wurden, innerhalb von fünf Jahren keinen Rückfall gegeben hat.

71 Nedopil, NStZ 2002, 344, 346.

72 M. Alex, Nachträgliche Sicherungsverwahrung - ein rechtsstaatliches und kriminalpolitisches Debakel, Diss. Bochum 2010, S. 93 m. Bespr. Sobota, GA 2011, 190; Kinzig, Die Legalbewährung gefährlicher Rückfalltäter, 2008, S. 196 ff.; Auch Dessecker kommt in „Lebenslange Freiheitsstrafe und Sicherungsverwahrung“, KrimZ 2011, S 34 zu dem Ergebnis, dass die Legalbewährung ehemals Sicherungsverwahrter erstaunlicherweise deutlich günstiger ausfällt als nach einer Entlassung aus dem Vollzug der Freiheitsstrafe. Zuvor gab es bereits ähnliche Befunde aus den USA, sogar für die Gruppe der als noch gefährlicher eingeschätzten psychisch Kranken: „Baxstrom“ 1966, „Dixon“ 1971 - Überblick m.w.N. bei Kinzig, Die Sicherungsverwahrung auf dem Prüfstand, Freiburg 1996, S. 87 ff.

73 Schöch empfindet diese Befunde in GA 2012, 14, 30 als „erschütternd“, obwohl er dem $B$ VerfG zuvor „verdienstvolle“ Ausführungen zu den Qualitätsanforderungen an eine Kriminalprognose (2004) bescheinigt. Streng in JZ 2011, 827, 828 lassen die Ergebnisse von $M$. Alex „ernüchtern“.

74 I.d.R., weil der nun diagnostizierten Gefährlichkeit keine neuen Tatsachen (sog. nova) zu Grunde lagen, M. Alex, wie Fn. 72, S. 89.

75 M. Alex, wie Fn. 72, S. 93.

76 \$66b StGB: „Erhebliche Straftaten [...], durch welche die Opfer seelisch oder körperlich schwer geschädigt werden".

77 Auch Nedopil, NStZ 2002, 344, 348 betont die Zeit als wesentliche Grenze prognostischer Sicherheit. Zusätzlich erschwert werde die Prognose bei der Klientel der Sicherungsverwahrung durch die Seltenheit der vorherzusagenden Ereignisse.

78 BVerfG, NJW 2009, 980, 983.

79 So bereits Sobota, GA 2011, 190, 191.

80 Anders die fragwürdige Auffassung der bayerische Justizministerin Merk in der Pressemitteilung149/08 vom 11.09.2008, zitiert nach Alex, wie Fn. 72, S. 116: „[...] Auch wer im fünften Jahr wieder ein Gewaltdelikt begeht, hat sich als gefährlich erwiesen!“

81 Schöch in FS Roxin II, S. 1193, 1213.

82 BVerfGE 128, 326, 399.

83 Diese Frage stellte auch schon Eschelbach in NJW 2010, 2500. Diesbezüglich würde auch eine stark verbesserte Kriminalprognostik nicht weiterhelfen, solange sie nicht $100 \%$ Treffgenauigkeit bieten kann.

84 BVerfGE 109, 133, 158. Dazu passt auch die regelmäßig schematische Abwägung zu Lasten der individuellen Freiheit der (diesmal konventionswidrig) Inhaftierten z.B. in BVerfG 2 BvR 769/10 - dazu beißende Kritik („Karlsruher Landrecht") von Pollähne in KJ 2010, 254 m.w.N.; a.A. Streng, JZ 2011, 827, 828

85 Und anders als bspw. bei der üblichen Gefahrenabwehr nach POG droht bei der Sicherungsverwahrung der potentiell endgültige Verlust der persönlichen Freiheit.

86 A.A. ohne jede Begründung: BVerfGE 109, $133,152$.

87 Streng, JZ 2011, 827, 830 mit Verweis auf Stree, Deliktsfolgen und Grundgesetz, 1960, S. $221 \mathrm{f}, 224$.

88 BVerfGE 109, 133, 150 m.w.N.
89 Schöch, GA 2012, 14, 31.

90 Vgl. nur Sch/Sch-Stree/Kinzig $\$ 57$ StGB Rn. 9.

91 Trotz des Unsinns einer solchen Kombination (Schöch, GA 2012, 14, 15: „überflüssig und nur symbolisch“) häufen sich zuletzt die Urteile, in denen lebenslange Haft mit Sicherungsverwahrung kombiniert wird: S. becklink 1018966 (sog. „Maskenmann“); becklink 1014335 u. 1018316 (sog. „Doppelmord von Bodenfelde": hier sogar lebenslang, Sicherungsverwahrung und psychiatrisches Krankenhaus); http://www.wiesbadener-kurier.de/nachrichten/ vermischtes/11548825.html (sog. „Schlachthof-Mord“); becklink 1018544 (sog. „Parkplatzmörder“); becklink 254305 (Fall „Mitja“) usw. usf. Hier stellt sich die grundsätzliche Frage, ob ein solches Urteil als sanktionsrechtlicher „Overkill“ noch (im engeren Sinne) verhältnismäßig ist.

92 S.o. Fn. 72

93 Radtke, GA 2011, 636, 639.

94 Ablehnend Radtke, GA 2011, 636, 639, 649, der bei seinem Plädoyer für die Zweispurigkeit allerdings offenkundig unterstellt, dass „Gefährlichkeit" ohne weiteres feststellbar sei.

95 S.o. Fn. 62.

96 So etwa Kreuzer, NStZ 2010, 473, 479.

97 S. Pressemitteilung des Bundesministeriums der Justiz vom 07.03.2012, abrufbar unter http:// www.bmj.de.

98 N. Leygraf, Stellungnahme vor dem Rechtsausschuss, S. 6, abrufbar unter www.bundestag.de.

99 Vgl. nämlich die Mitteilung „Straßburger Lob für das höchste deutsche Gericht" in Legal Tribune ONLINE vom 09.06.2011 zu den Urteilen vom 09.06.2011, Beschwerdenummern 30493/04 - S. gegen Deutschland und 31047/04, 43386/08 - M. gegen Deutschland, wonach der EGMR ausdrücklich den deutschen Ansatz, „die Bestimmungen des Grundgesetzes auch im Licht der Konvention und der Rechtsprechung des EGMR auszulegen, mit dem das Verfassungsgericht sein fortwährendes Bekenntnis zum Grundrechtsschutz nicht nur auf innerstaatlicher, sondern auch auf europäischer Ebene unterstreicht", begrüßt.

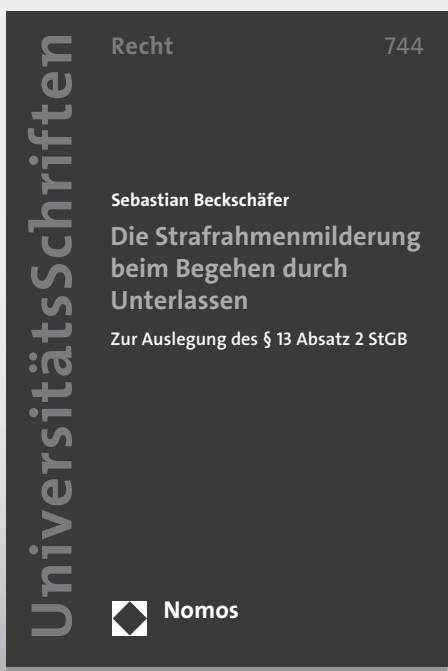

\section{Die Strafrahmenmilderung beim Begehen durch Unterlassen}

Zur Auslegung des $§ 13$ Absatz 2 StGB

Von Sebastian Beckschäfer

2012, ca. 136 S., brosch., ca. 39,- €, ISBN 978-3-8329-7404-6

(Nomos Universitätsschriften - Recht, Bd. 774)

Erscheint ca. Oktober 2012

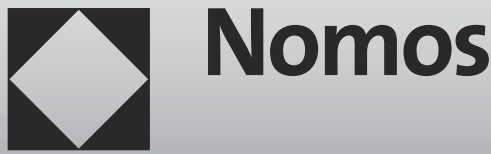

